\title{
Prurito
}

\section{Consigli pratici di gestione nei pazienti eleggibili alle cure palliative pediatriche}

\section{Irene Avagnina, Serena Condemi, Chiara Paolin, Franca Benini}

Centro Regionale Veneto di Terapia del Dolore e Cure Palliative Pediatriche, Dipartimento di Salute della Donna e del Bambino, Università di Padova

\section{Introduzione}

Le cure palliative pediatriche (CPP) rappresentano un ambito multidisciplinare della pediatria che si prefigge l'obiettivo di garantire la qualità di vita a bambini affetti da malattie inguaribili ad alta complessità assistenziale e di rispondere ai loro bisogni e a quelli delle loro famiglie.

A livello clinico, molteplici sono i sintomi disturbanti con ampia ricaduta sulla qualità della vita, che devono essere gestiti e trattati. Alcuni di questi in modo particolare rappresentano una sfida importante per i caregiver e i sanitari che si occupano dei bambini eleggibili alle CPP.

Il prurito e i disturbi del sonno sono frequenti e invaldanti in $\mathrm{CPP}$ e possono determinare un impatto fortemente negativo sulla qualità di vita del bambino e possono risultare difficili da gestire per i clinici.

\section{Prurito}

\section{Definizione e cause}

Il prurito è definito come una sensazione cutanea spiacevole che provoca l'urgenza di grattarsi.

Il prurito può rappresentare un sintomo estremamente invalidante, che impatta negativamente sulla qualità della vita del bambino andando a compromettere la qualità del sonno e lo svolgimento della attività quotidiane. Inoltre, il prurito può portare a lesioni cutanee con possibilità di sovrainfezioni.

Nei pazienti in cure palliative pediatriche il prurito è un sintomo relativamente frequente, soprattutto nel fine vita, e la sua genesi nella maggior parte dei casi è multifattoriale.

Le cause principali di prurito in CPP sono: cause iatrogene, insufficienza renale cronica, colestasi epatica, patologie o comorbidità dermatologiche, disordini ematologici o linfoproliferativi o altre forme tumorali, disturbi psicologici, HIV, oltre alle più comuni cause di prurito in pediatria come xerosi cutanea, dermatite atopica, eczemi, orticaria o altre dermatiti cutanee, infezioni cutanee (scabbia, parassitosi).

Di seguito vengono descritte le cause più frequenti in CPP.

- Prurito uremico. Sintomo frequente associato a insufficienza renale cronica avanzata o terminale, soprattutto in pazienti in dialisi. Può presentarsi anche in pazienti con insufficienza renale secondaria, per esempio da scompenso cardiaco. Il prurito in questi casi può essere intermittente o continuo.

- Prurito da colestasi. Esso è più frequente nel paziente oncologico con ostruzione biliare extraepatica e/o intraepatica. In questi casi l'incidenza del prurito è alta (circa nel $45 \%$ dei casi).

- Prurito in patologie dermatologiche. Nel contesto delle cure palliative pediatriche, delle patologie dermatologiche primarie associate a prurito, l'epidermolisi bollosa è sicuramente la più severa e di difficile gestione. Tuttavia, sempre in CPP, il riscontro di lesioni cutanee secondarie o infezioni cutanee è frequente e può associarsi a prurito.
- Prurito farmaco-correlato. Gli oppioidi sono la causa più comune di prurito farmaco-correlato nei pazienti in cure palliative pediatriche. Inoltre, alcuni antibiotici, come penicilline, trimetoprim-sulfametoxazolo, alcuni antiepilettici come la fenitoina, FANS e alcuni farmaci antitumorali e biologici sono stati associati a prurito anche in assenza di eruzione cutanee.

In corso di terapia oppioide, il prurito è stato riportato in circa l'ı\% dei casi. Forme invece transitorie di prurito sono riportate nel circa Io\% di pazienti in terapia oppioide cronica. Quando presente il prurito è generalizzato e non associato a rash cutanei. In genere il prurito è più comune con l'uso di oppioidi naturali, come codeina e morfina, rispetto agli oppioidi semisintetici (idrossicodone e ossicodone) o sintetici (metadone e fentanyl), anche se i dati in letteratura sono abbastanza discordanti.

- Tumori maligni. Diverse forme di neoplasie maligne, anche non associate a colestasi, possono causare prurito. I disturbi maligni ematologici e non ematologici più comuni associati al prurito includono linfoma di Hodgkin, linfoma non Hodp gkin, sindromi mieloproliferative come la policitemia vera, micosi fungoide, leucemie e tumori carcinoidi gastrici produttori di istamina. In generale, la prevalenza del prurito è maggiore nelle neoplasie ematologiche rispetto ai tumori solidi. Nella maggior parte dei casi, in queste situazioni il prurito è da correlarsi a una sindrome paraneoplastica; meno frequentemente è legato a metastasi cutanee.

\section{Sintomatologia}

La sintomatologia è caratterizzata da una sensazione "fastidiosa" e talvolta molto "urgente" che stimola in maniera volontaria e/o automatica il grattarsi. Lo stimolo meccanico determinato dal grattamento innesca un circuito inibitorio sulla trasmissione stessa dello stimolo pruriginoso limitando così la sintomatologia.

Il prurito non si associa sempre a lesioni o alterazioni cutanee, ma come complicanza si possono osservare lesioni da grattamento e sovrainfezioni cutanee.

\section{Diagnosi}

La diagnosi del prurito è clinica. L'approfondimento anamnestico è volto all'individuazione delle cause.

A livello anamnestico vanno indagati:

- localizzazione (localizzato o sistemico), intensità, durata, frequenza degli episodi di prurito;

- fattori precipitanti o allevianti;

- percezione soggettiva del sintomo;

- impatto sulla qualità di vita;

- stato nutrizionale e di idratazione;

- storia di viaggi recenti;

- anamnesi farmacologica.

L'esame obiettivo deve comprendere un'attenta valutazione di tutta la cute per escludere presenza di rash, lesioni o altre manifestazioni cutanee. Inoltre un esame obiettivo completo può aiutare il clinico nell'inquadramento diagnostico del prurito (per esempio per l'individuazione di epatosplenomegalia nelle forme colestatiche o di linfoadenomegalia nelle malattie linfoproliferative).

Esami strumentali o ematochimici sono indicati in funzione del miglior inquadramento o monitoraggio delle cause sottostanti.

\section{Trattamento}

Il trattamento del prurito prevede simultaneamente la correzione delle cause (ove possibile) e il trattamento del sintomo. Per quanto concerne il trattamento del prurito le strategie possono essere ulteriormente suddivise in farmacologiche e non farmacologiche. 
Tabella 1

\begin{tabular}{|c|c|c|}
\hline Cause & Fisiopatologia & Trattamento \\
\hline Prurito da oppioidi & $\begin{array}{l}\text { Il prurito è secondario all'attiva- } \\
\text { zione dei recettori } \mu \text { (meccanismo } \\
\text { non istamino mediato). } \\
\text { Gli oppiacei possono indurre una } \\
\text { reazione serotoninergica seconda- } \\
\text { ria al prurito. }\end{array}$ & $\begin{array}{l}\text { - Rotazione dell'oppioide. } \\
\text { - Antistaminico (idrossizina). } \\
\text { - Naloxone a basse dosi (0,25-2 mcg/kg/h) con morfina ic. } \\
\text { - Antagonisti serotoninergici come l'ondansetron (0,15-0,2 mg/ } \\
\text { kg max } 8 \text { mg dose). } \\
\text { - FANS, propofol (nell'end of life), antagonisti dopaminergici } \\
\text { selettivi D2 (aloperidolo), rifampicina. } \\
\text { - NON raccomandata profilassi del prurito. }\end{array}$ \\
\hline
\end{tabular}

Prurito uremico

Patogenesi del prurito poco Lieve:

nota, possibilmente associata a - Terapia non farmacologica e pomate topiche.

fattori pro-infiammatori, citochine, - Antistaminici.

incremento dei livelli plasmatici di Moderato/servero:

istamina, carenza marziale. $\quad$ - UVB fototerapia.

- Antidepressivi come sertralina o mirtazapina.

- Acido gamma linoleico crema x 2 v/die.

- Agopuntura.

- Pomate a base di inibitori topici della calcineurina.

- Intensificazione dialisi.

- Gabapentin (solo nei pazienti dializzati o nell'end of life data la sua nefrotossicità).

\section{Prurito da colestasi}

Prurito da patologia linfoproliferativa
II prurito è secondario al deposito cutaneo di acidi biliari e all'incremento dei neurotrasmettitori endogeni degli oppioidi.
Lieve:

- Terapia non farmacologica e pomate topiche.

- Antistaminici.

- Lieve/moderato.

- Colestiramina.

- Acido ursodesossicolico.

Moderato/severo:

- Rifampicina.

- Fenobarbital.

- Naloxone basse dosi.

- Ondansetron.

- Corticosteroidi.

- Antidepressivi come sertralina e mirtazapina.

- Stent del dotto in caso di refrattarietà.

- NON efficaci gabapentinoidi, antistaminici.
La patofisiologia del prurito non è nota, sembra verosimilmente di natura pro-infiammatoria.
Lieve:

- Supporto non farmacologico.

- Antistaminici (idrossizina).

- Pomata sodio cromoglicato 5\%.

Lieve/moderato:

- Gabapentin a basse dosi (15 mg/kg/die in 3 dosi) con inizio graduale.

- Antidepressivi a basse dosi (sertralina, mirtazapina...).

Moderato/severo:

- Corticosteroidi.

- UV fototerapia.

- Pomate a base di inibitori topici della calcineurina nelle GVHD cutanee.

- Talidomide.
Prurito da epidermolisi bollosa
Meccanismo multifattoriale legato al danno cutaneo, al dolore cronico e a fattori iatrogeni (medicazioni, disinfezioni, terapia oppioide).
- Ansiolisi e premedicazione antalgica prima del bagno o di manovre dolorose.

- Gabapentin a basse dosi (15 mg/kg/die in 3 dosi) con inizio graduale.

- Farmaci biologici (dupilumab).

- Antistaminici.

- Corticosteroidi.

- Antidepressivi (amitriptilina, sertralina).

- Ondansetron.

- Bagni di farina di avena (beneficio aneddotico) 
Il trattamento non farmacologico prevede:

- educazione genitoriale all'igiene e all'adeguata idratazione cutanea con creme emollienti topiche, senza profumi o altri allergeni;

- mantenere le unghie sempre corte e pulite per evitare lesioni da grattamento o/e sovrainfezioni; in casi estremi consigliato l'utilizzo di guanti o muffole;

- mantenere l'ambiente fresco e umidificato;

- utilizzare abiti di cotone e lavare gli indumenti con detergenti naturali o senza allergeni;

- garantire al paziente un adeguato stato nutrizionale e di idratazione;

- per quanto concerne il sollievo acuto dal prurito, esso si riduce con l'applicazione di freddo, con la distrazione e il rilassamento;

- evitare situazioni che inducano vasodilatazione come esposizione al calore, consumo di alcol o caffeina.

Il trattamento farmacologico sintomatico del prurito prevede l'utilizzo di strategie topiche e sistemiche. Per quanto concerne i trattamenti topici vengono utilizzate:

- pomate rinfrescanti (I\% mentolo o 0,5-2\% canfora);

- pomata alla calamina oleosa;

- Caladry $1^{\circledR}$ crema 2 applicazioni/die;

- pomate steroidee in caso di segni di infiammazione/allergia (dermatiti, eczemi, atopia);

- pomata antibiotica in caso di sovrainfezione;

- pomate alla capsaicina a basse dosi (0,025-0,075\%); efficacia aneddotica e poco tollerata nel bambino;

- pomate anestetiche a base di lidocaina, in caso di prurito localizzato (in questo caso tuttavia va prestata attenzione all'estensione dell'area di utilizzo e alla frequenza, dati i possibili effetti sistemici della lidocaina);

- pomate a base di inibitori topici della calcineurina, inclusi tacrolimus (0,03 e ০,I\% unguenti) e pimecrolimus (I\% crema), indicati nel prurito uremico o in quello da dermatite atopica, nel prurito anogenitale e nel prurito da GVHD cutanea;

- creme emolienti per l'idratazione cutanea che solitamente vengono applicate intervallandole alle pomate specifiche per il prurito.

Per quanto concerne il trattamento sistemico del prurito i farmaci più comunemente utilizzate in cure palliative pediatriche includono antagonisti del recettore 5 - $\mathrm{HT}_{3}$; antagonisti degli oppioidi; agonisti/ antagonisti misti dei recettori $\mu$; antidepressivi come gli SSRI sertralina, paroxetina e mirtazapina; anticonvulsivanti; farmaci antiinfiammatori non steroidei (FANS); rifampicina e antagonisti del recettore della neurochinina I (NKI).

In precedenza, gli antistaminici (antagonisti del recettore $\mathrm{HI}$ ) erano i farmaci di scelta raccomandati per qualsiasi tipo di prurito. Tuttavia, poiché la maggior parte delle cause del prurito nelle cure palliative pediatriche non comporta il rilascio di istamina, questa pratica non è più raccomandata, a meno che un componente dell'istamina non sia chiaramente coinvolto nel prurito. Tuttavia l'effetto sedativo degli antistaminici può avere comunque una ricaduta benefica nei pazienti che non sono in grado di dormire a causa dei sintomi di prurito; in questi l'antisitaminico consigliato è l'idrossizina ( $\mathrm{I}-2 \mathrm{mg} / \mathrm{kg} /$ die dalla mono-somministrazione serale fino a $4 \mathrm{v} / \mathrm{die}$ ).

Per la maggior parte dei pazienti con prurito, di solito il trattamento viene definito rispetto alla causa sottostante e si inizia con una classe di farmaci; se non c'è sollievo entro 24-48 ore, si passa a una classe farmacologica diversa.

Nella Tabella 1 vengono riassunte alcune strategie terapeutiche specifiche per la gestione del prurito.

Infine, più raramente vengono utilizzate altre strategie quali:

- stimolazione elettrica transcutanea (TENS), utilizzata soprattutto in caso di prurito localizzato (per esempio nelle masse fungoidi);
- agopuntura;

- fototerapia nel prurito uremico o da patologia linfoproliferativa;

- reflessologia e tecniche di rilassamento.

Take home message

- Il prurito è un sintomo potenzialmente invalidante e spesso difficile da gestire.

- Il trattamento del prurito deve essere personalizzato in relazione alla fisiopatologia sottostante il sintomo e alla sua gravità.

\section{Caso clinico}

Oliver, Io anni, affetto da epidermolisi bollosa, lamenta importante dolore, esacerbato soprattutto dai cambi della medicazione e dall'igiene, e prurito.

Per il trattamento antalgico, in considerazione del potenziale effetto pruritogeno di alcuni oppioidi, è stata consigliata una terapia fissa con metadone e un'ansiolisi con benzodiazepine prima della care.

Con tali strategie è stato osservato un miglioramento del controllo del dolore ma non del prurito.

In accordo con lo specialista dermatologo, data la refrattarietà del sintomo, è stata proposta una terapia con dupilumab, che ha dimostrato efficacia in letteratura per la dermatite atopica; attualmente è in corso di studio per l'epidermolisi bollosa, con risultati promettenti.

\section{Bibliografia}

1. R. Hain. Palliative Care for Children. Oxford Textbook, 202I (3).

2. Kang SY, Um JY, Chung BY, et al. Differential Diagnosis and Treatment of Itching in Children and Adolescents. Biomedicines. 202I Jul 30;9(8):919.

3. Zhukovsky DS, Rozmus CL, Robert RS, et al. Symptom profiles in children with advanced cancer: Patient, family caregiver, and oncologist ratings. Cancer. 20I5 Nov I5;I2I(22):4080-4087.

4. He F, Jiang Y, Li L. The effect of naloxone treatment on opioid-induced side effects: A meta-analysis of randomized and controlled trails. Medicine (Baltimore). 20I6 Sep;95(37):e4729.

5. Gurnani P, Miloh T, Chandar J, et al. Systemic causes of non-dermatologic chronic pruritus in the pediatric population and their management: An unexplored area. Pediatr Dermatol. 202I Sep;38(5):IO5I-IO60.

6. Papanikolaou M, Onoufriadis A, Mellerio JE, et al. Prevalence, pathophysiology and management of itch in epidermolysis bullosa. Br J Dermatol. 202I May;I84(5):816-825. 